\title{
Real-time in vivo rectal wall dosimetry using MOSkin detectors during linac based stereotactic radiotherapy with rectal displacement
}

Kimberley Legge ${ }^{1 *}$ D, Peter B. Greer ${ }^{1,2}$, Daryl J. O'Connor ${ }^{1}$, Lee Wilton ${ }^{2}$, Matthew Richardson ${ }^{2}$, Perry Hunter ${ }^{2}$, Alex Wilfert ${ }^{2}$, Jarad Martin ${ }^{2}$, Anatoly Rosenfeld ${ }^{3}$ and Dean Cutajar ${ }^{3}$

\begin{abstract}
Background: MOSFET dosimetry is a method that has been used to measure in-vivo doses during brachytherapy treatments and during linac based radiotherapy treatment. Rectal displacement devices (RDDs) allow for safe dose escalation for prostate cancer treatment. This study used dual MOSkin detectors to assess real-time in vivo rectal wall dose in patients with an RDD in place during a high dose prostate stereotactic body radiation therapy (SBRT) boost trial.

Methods: The PROMETHEUS study commenced in 2014 and provides a prostate SBRT boost dose with a RDD in place. Twelve patients received two boost fractions of 9.5-10 Gy each delivered to the prostate with a dual arc volumetric modulated arc therapy (VMAT) technique. Two MOSkins in a face-to-face arrangement (dual MOSkin) were used to decrease angular dependence. A dual MOSkin was attached to the anterior surface of the Rectafix and read out at $1 \mathrm{~Hz}$ during each treatment. The planned dose at each measurement point was exported from the planning system and compared with the measured dose. The root mean square error normalised to the total planned dose was calculated for each measurement point and treatment arc for the entire course of treatment.

Results: The average difference between the measured and planned doses over the whole course of treatment for all arcs measured was $9.7 \%$ with a standard deviation of $3.6 \%$. The cumulative MOSkin reading was lower than the total planned dose for $64 \%$ of the arcs measured. The average difference between the final measured and final planned doses for all arcs measured was 3.4\% of the final planned dose, with a standard deviation of $10.3 \%$.

Conclusions: MOSkin detectors were an effective tool for measuring dose delivered to the anterior rectal wall in real time during prostate SBRT boost treatments for the purpose of both ensuring the rectal doses remain within acceptable limits during the treatment and for the verification of final rectal doses.
\end{abstract}

Keywords: Prostate cancer, MOSFET detector, In vivo dosimetry, Stereotactic radiation therapy

\footnotetext{
* Correspondence: Kimberley.Legge@uon.edu.au

'University of Newcastle, University Drive, Callaghan 2308, NSW, Australia

Full list of author information is available at the end of the article
} 


\section{Background}

Prostate cancer shows a radiation response consistent with a low $\alpha / \beta$ ratio, indicating that hypofractionated treatment schedules may increase the effectiveness of treatment [1, 2]. Hypofractionation includes the delivery of higher dose fractions to boost the total dose prior to or following conventionally fractionated treatment. Delivery of a boost dose using high dose rate (HDR) brachytherapy in combination with conventionally fractionated external beam radiation therapy (EBRT) has been shown to improve tumour control rates when compared with conventionally fractionated EBRT alone [35]. EBRT is far more accessible than brachytherapy in Australia, and low rates of rectal and urinary toxicity along with good biological response rates have been observed in patients who received a boost dose delivered using an SBRT technique [6-8].

Rectal complications can arise as a result of radiation therapy for prostate cancer, and delivery of higher doses through hypofractionation increases the risk of damage to surrounding healthy tissues [9]. The risk and severity of rectal toxicities has been correlated with the volume of rectal wall exposed to high doses of radiation [10]. Use of dose volume constraints in planning and daily imaging to enable reduced margins are the most effective ways to reduce rectal dose, but rectal sparing devices such as injected spacer materials and the Rectafix rectal retractor have also been used to allow for safe dose escalation [11]. The Rectafix system (Fig. 1) uses a rod which is inserted into the patient's rectum. The rod is then moved posteriorly on the vertical column and locked in place to manually move the rectum away from the prostate. The vertical column is marked to allow reproducible retraction. The Rectafix has been found to provide an average increase in separation of $0.5 \mathrm{~cm}$ and to assist in immobilising the rectal wall by preventing changes in filling by gas or faeces [12].
Changes in bladder or rectal volume during treatment can affect the dose coverage of the prostate and increase the dose to healthy tissues. One way to monitor the effect of any motion is real-time in-vivo dosimetric monitoring during treatment. Previous studies in this area have used plastic scintillation detectors and MOSFET based dosimeters in phantoms [13-16]. Plastic scintillation detectors attached to an endorectal balloon inserted in an anthropomorphic prostate phantom were used by Archambault et. al. to provide dose measurements every $150 \mathrm{~ms}$ during eight field intensity modulated radiation therapy treatments. In a field by field comparison, plastic scintillation detectors were found to agree with treatment planning system doses to within $0.5 \%$ [13]. MOSkin detectors are MOSFET based dosimeters. They have successfully been used to measure urethral dose inside a catheter placed within a gel phantom simulating a typical HDR brachytherapy treatment, with two MOSkin detectors used in a face to face arrangement (a dual MOSkin) found to be more accurate than a single MOSkin detector [14]. Dose to the anterior rectal wall in a phantom study of tomotherapy prostate boost dose while attached to a replica Rectafix rod has also been verified with MOSkins, with all dual MOSkins used in this study agreeing with treatment planning system dose to within $\pm 5 \%$ when placed in a high dose gradient region [15]. MOSkins have been integrated into a transrectal ultrasound probe of the kind used to guide interstitial needle insertion during HDR brachytherapy treatment. MOSkin measurements taken during three HDR brachytherapy treatment deliveries to a phantom resulted in an average discrepancy of $-0.6 \pm 2.6 \%$ between the measured and planned doses [16].

There have been very limited in-vivo patient dosimetry studies, however, in-vivo measurements have been performed during HDR brachytherapy treatments and EBRT treatments. Plastic scintillation detectors attached

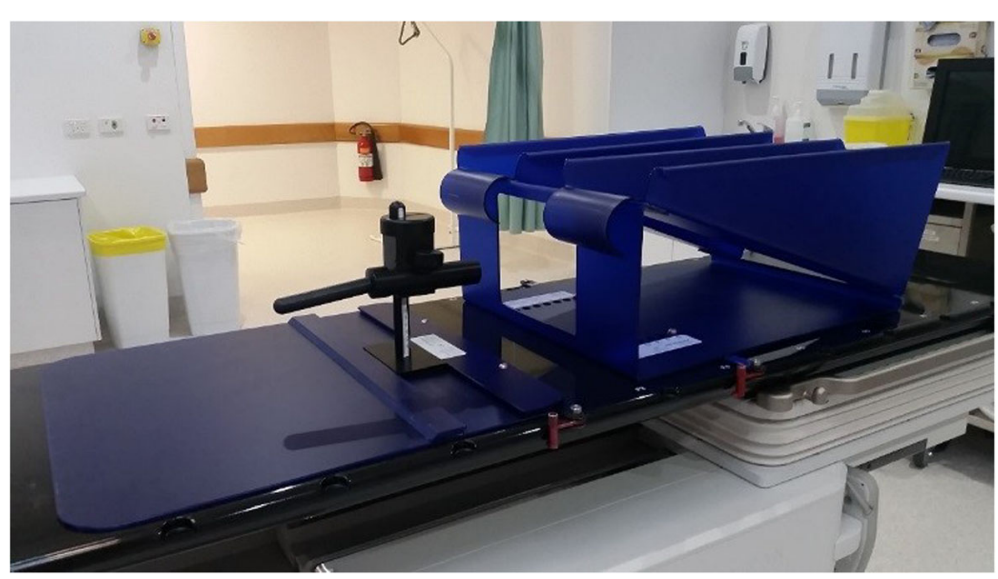

Fig. 1 The Rectafix system 
to an endorectal balloon were used by Wootton et al. to measure dose to the rectal wall over 142 prostate EBRT fractions for five patients. The average difference between measured doses and doses calculated on day of treatment CT scans was $-0.4 \pm 2.8 \%$ [17]. In a study of 12 patients by Carrara et. al., MOSkins integrated into a transrectal ultrasound probe were shown to accurately measure dose delivered to the rectal wall during HDR brachytherapy treatments. The MOSkin measured doses were found to align more closely with delivered doses reconstructed after treatment than with planned doses in this case. The average absolute difference between MOSkin measured doses and reconstructed doses was $3.6 \pm 1.9 \%$, while the difference between MOSkin measured doses and planned doses was $6.7 \pm 5.1 \%$, with a total measurement uncertainty of $5.7 \%$, demonstrating their potential for use in detecting treatment delivery errors [18]. However, assessment of rectal dose delivered during EBRT boost treatments using MOSkin detectors within patients has not been investigated to date, and previous studies have only compared measured doses with total planned doses for each field or treatment.

The aim of this work was to examine the feasibility of determining real-time in vivo rectal wall dose during prostate SBRT with a rectal retractor by monitoring during treatment with a dual MOSkin detector. Measured doses were compared with planned doses across the whole course of each treatment arc delivered to provide a measure of the accuracy of delivery, including treatment plan dose calculation and patient setup errors.

\section{Methods}

1. Patient and treatment details The PROstate Multicentre External beam radioTHErapy Using Stereotactic boost (PROMETHEUS) study commenced in late 2014 and provides an EBRT boost dose consisting of two fractions to patients prior to a conventionally fractionated course of EBRT. A Rectafix rectal sparing device was in place during both boost fractions for the patients in this study. Twelve patients with intermediate to high risk prostate cancer (median age 72) consented to the ethics board approved study. EBRT boost treatments were planned using the Eclipse treatment planning system (version 11) and were delivered using either a Varian Clinac iX linear accelerator with a $6 \mathrm{MV}$ photon beam at a maximum dose rate of $600 \mathrm{MU} / \mathrm{min}$ or using a Varian Truebeam linear accelerator with a 10 MV beam with a maximum dose rate of $600 \mathrm{MU} /$ min or $10 \mathrm{MV}$ flattening filter free photon beam with a maximum dose rate of $2400 \mathrm{MU} / \mathrm{min}$. Six patients received a boost dose of 9.5 Gy per fraction and six patients received a boost dose of 10 Gy per fraction. All patients were treated with a Rectafix system in place. The Rectafix system is shown in Fig. 1. Patients received a pre-treatment cone beam computed tomography (CBCT) scan to check Rectafix positioning and bladder and rectal filling and were aligned using implanted gold fiducial markers. The prostate position in patients treated on the Truebeam was monitored using triggered two dimensional kilovoltage images taken every $3 \mathrm{~s}$ during treatment. Mid-treatment CBCT scans for repositioning were taken for all patients treated on the Clinac, and for patients treated on the Truebeam for whom the triggered imaging indicated prostate displacement had occurred. All patients received a post-treatment CBCT scan.

2. MOSkin Design

MOSkin detectors, developed at the Centre for Medical Radiation Physics, University of Wollongong, are MOSFET based detectors designed to measure dose at the air-skin interface and have an active volume of $4.8 \times 10^{-6} \mathrm{~mm}^{3}$ with a gate oxide thickness of $0.55 \mu \mathrm{m}$ [19] and a water equivalent depth of $0.07 \mathrm{~mm}$. This small volume makes them desirable for measurements in high dose gradient regions, [20] such as the anterior rectal wall in a prostate SBRT boost plan. MOSkin detectors provide temperature independent measurements between 15 and $40{ }^{\circ} \mathrm{C}$ [21] and have reproducible sensitivity for fraction sizes up to $10 \mathrm{~Gy}$ [20]. Two MOSkins in a face-to-face arrangement (a dual MOSkin) have been found to reduce angular dependence resulting from the geometrical construction of the detector [22]. The dual MOSkin threshold voltage can be read out at $1 \mathrm{~Hz}$ and real-time readout software developed by the University of Wollongong can be used to convert the voltage to dose and display the cumulative dose delivered to the MOSkin as treatment progresses. MOSkin detectors are visible on cone beam CT (CBCT) scans. The MOSkin readout unit a dual MOSkin attached to the Rectafix rod appear in Fig. 2.

3. Calibration and Testing

Prior to treatment using the linear accelerator, dual MOSkin detectors were calibrated at the energy and dose rate that was to be used for patient treatment. Dual MOSkins were aligned to isocentre using lasers and placed under $1.5 \mathrm{~cm}$ of solid water for $6 \mathrm{MV}$ treatments, and under $2.5 \mathrm{~cm}$ of solid water for 10 MV treatments, with a $100 \mathrm{~cm}$ source to surface distance, so that the MOSkin would lie at the depth of dose maximum. A field size of $10 \mathrm{~cm} \times 10 \mathrm{~cm}$ and collimator angle of $0^{\circ}$ was used. Calibration was performed by delivering $20 \mathrm{cGy}$, as determined by 


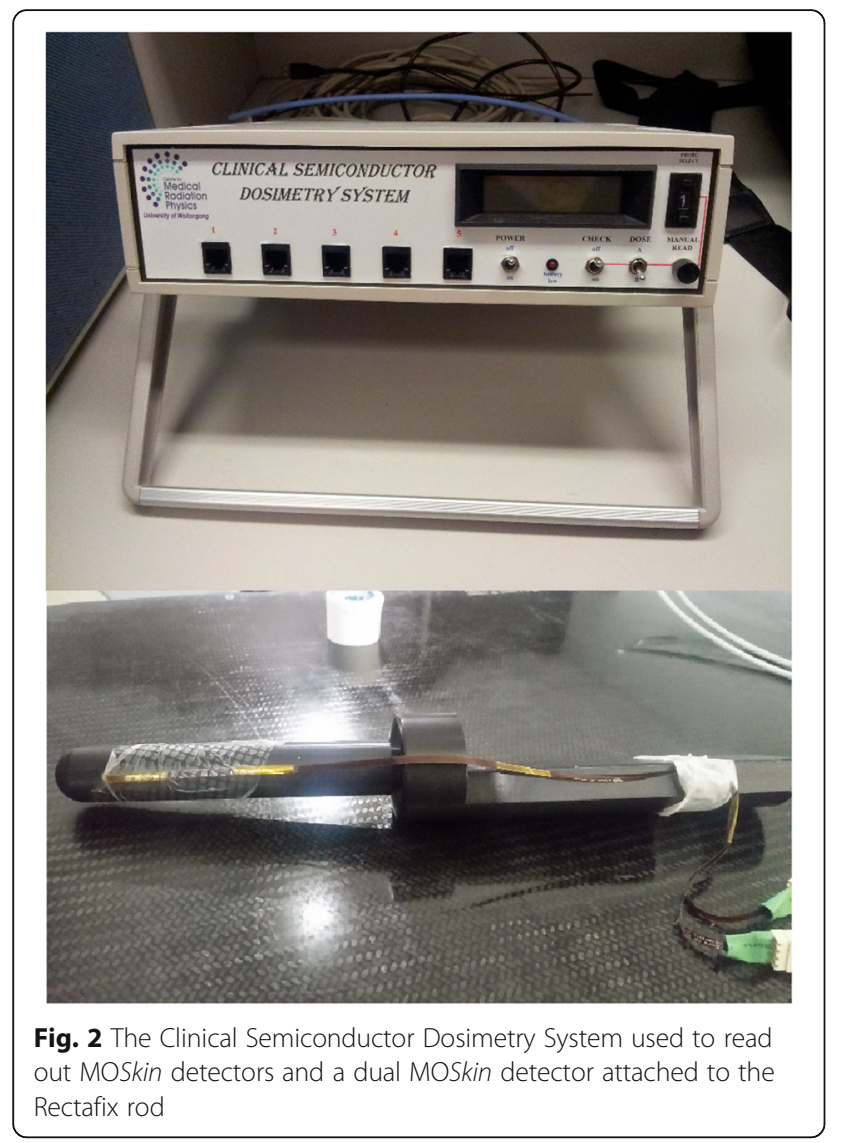

nominal machine output, to the dual MOSkin arrangement in five separate irradiations. The dual MOSkin was then inverted, placing both detectors in inverse orientation so that the other detector was closest to the beam, and another five irradiations of 20 cGy were delivered.

To determine a calibration factor for each dual MOSkin, the reading for each individual MOSkin detector at $30 \mathrm{~s}$ after beam off was recorded for each delivery, resulting in 20 total voltage values, five from each detector $(\times 2$ detectors $)$ in the initial orientation and five from each detector in the inverse orientation. The 20 values obtained were then averaged. A calibration factor, $\mathrm{CF}$ (mV/cGy) was then obtained by dividing the average threshold voltage shift $\left(\Delta \mathrm{V}_{\mathrm{av}} \mathrm{mV}\right)$ by the delivered dose (20 cGy) as shown in Eq. 1.

$\boldsymbol{C F}=\frac{\Delta \boldsymbol{V}_{\boldsymbol{a v}} m V}{20 c G y}$

Dual MOSkin detectors were tested for angular dependence by irradiation in the centre of a solid water cylinder $20 \mathrm{~cm}$ in diameter. The cylinder was raised $5 \mathrm{~cm}$ from the couch using solid water blocks for supports. The dual MOSkin was rotated relative to the beam and irradiated after every $30^{\circ}$ rotation.

MOSkin detectors were also tested for dose linearity from 10 to 200 cGy and dose rate dependence from $100 \mathrm{MU} / \mathrm{min}$ to $600 \mathrm{MU} / \mathrm{min}$ at $1.5 \mathrm{~cm}$ depth in solid water using a Varian Clinac iX linear accelerator.

4. Phantom Study

As a final end-to-end test, a dual MOSkin was placed in a specially made insert in a CIRS 801-P Virtually Human Male Pelvis phantom (CIRS Inc., Norfolk, VA). A CT scan of the phantom with the insert in position was acquired and a 9.5 Gy dual arc VMAT plan was transferred to the CT scan and calculated. The treatment plan on the phantom with the specially made insert is shown in Fig. 3.

The phantom was aligned with CBCT using a phantom insert with fiducial markers. This insert was then carefully removed and the insert with a dual MOSkin device was placed in the phantom. The plan was delivered using a Varian Clinac iX linear accelerator and the dual MOSkin was read out at $1 \mathrm{~Hz}$ during delivery. The same steps as outlined below in part six were used to compare the MOSkin measured dose with the planned dose extracted from the treatment planning system.

5. Treatment Delivery

Prior to each treatment fraction, a dual MOSkin detector was attached to the anterior surface of the Rectafix rod using sterile adhesive wraps, as shown in Fig. 2. The entire rod was then covered with a condom to ensure that the risk of patient contact with the device was minimised. Each dual MOSkin was used for a single treatment fraction and then discarded. The location of the MOSkin was chosen by measuring within the Eclipse planning system, in the superior-inferior direction, the distance from the axial plane in which the collar of the rod appears to the axial plane in which the centre of the planning target volume (PTV) occurs so that the location was reproducible and as close to the centre of the PTV as possible.

The dual MOSkin was attached to the clinical semiconductor dosimetry system, which was attached to a laptop computer. Real-time readout software called FETch (Centre for Medical Radiation Physics, University of Wollongong) was used on the laptop for readout. The Rectafix, with attached dual MOSkin, was inserted into the patient prior to alignment. Detectors were inside the patient for over 10 min prior to irradiation, giving the detectors time to equalise with patient temperature. Detectors were zeroed immediately prior to treatment delivery and voltages read out from both detectors in the dual 


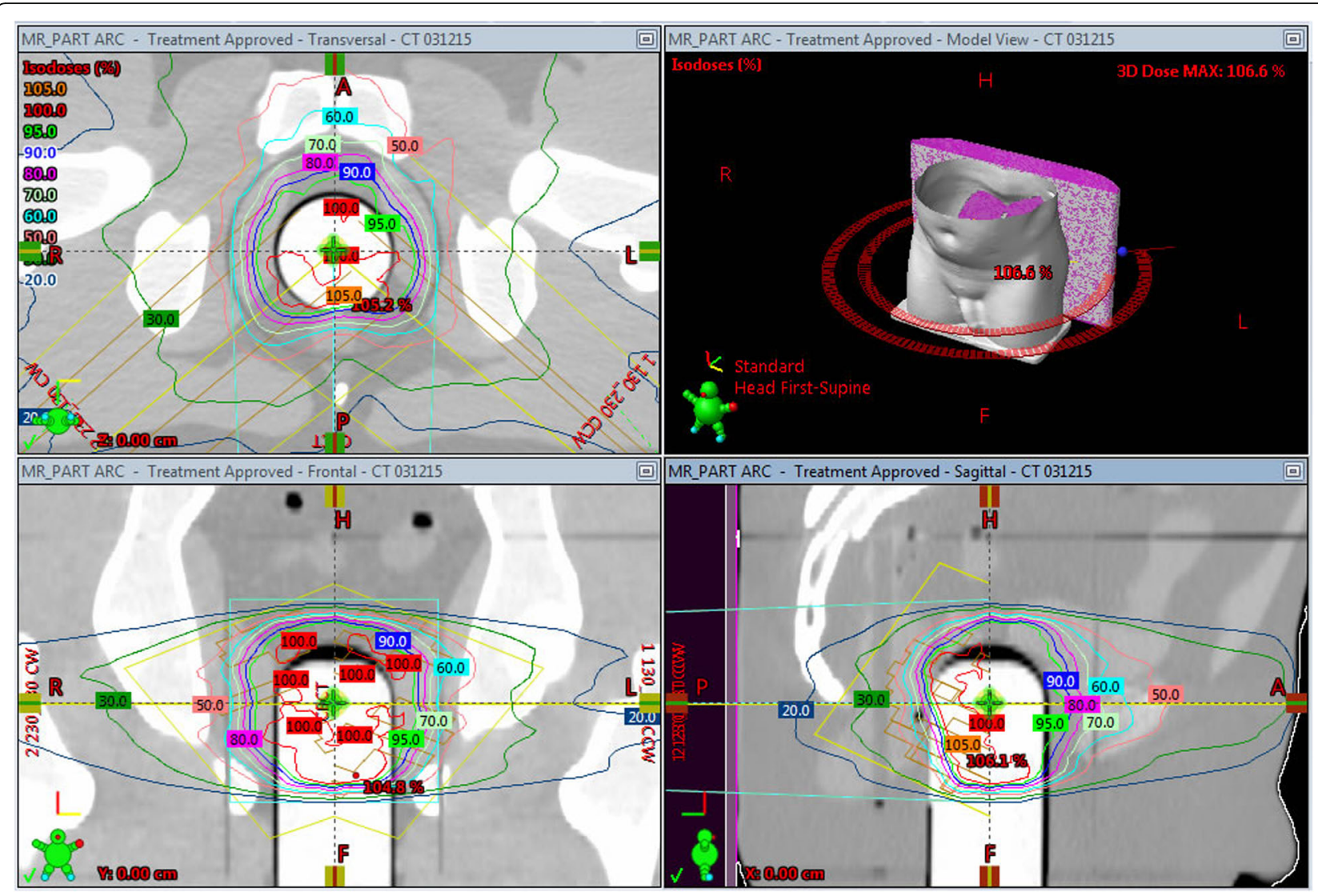

Fig. 3 Dose plan on phantom with specially made insert for dual MOSkin detector

MOSkin at $1 \mathrm{~Hz}$ during delivery of each two arc treatment. EPID images were acquired during treatment at $15.26 \mathrm{~Hz}$ using a research framegrabber computer. The gantry angle for each image was recorded by the imaging system so that the position of the gantry with time was known.

Following delivery, MOSkin readouts were imported into a spreadsheet. The doses recorded by each of the individual detectors making up the dual detector were averaged and the result divided by the detector's calibration factor.

6. Extraction of Planned Dose and Comparison Within the Eclipse treatment planning system, the location of the active area of the MOSFET in the $\mathrm{CBCT}$ acquired at the end of each fraction was marked. Each of these CBCTs was then manually registered to the patient's planning CT. A verification point was then placed on the planning $\mathrm{CT}$ at the location of the MOSFET, yielding two verification points per patient, one for each prostate SBRT boost fraction. Figure 4 shows a posttreatment $\mathrm{CBCT}$ scan of a patient with the active area of the MOSkin marked.
For patients treated using a $6 \mathrm{MV}$ or $10 \mathrm{MV}$ beam with a flattening filter, a verification plan was created in the Eclipse treatment planning system with the treatment split into sub-arcs, each of $5.2^{\circ}$. The dose file for each sub-arc and the plan file were then exported from the system. For $10 \mathrm{MV}$ flattening filter free beams, the version of the planning system available was unable to automatically split patient plans into sub-arcs. Five partial treatment plans were created for each of these patients, evenly splitting each arc by control points to obtain five cumulative dose points for each treatment. Following export of all dose and plan files, in-house software was used to extract the dose and gantry angle for each arc at the location of the MOSkin verification points. The position of the gantry with time was determined using spline interpolation from the gantry angles recorded in the header of the EPID images acquired at $15.26 \mathrm{~Hz}$ during treatment.

Planned dose at the verification point and MOSkin measured dose were then plotted as a function of time over the whole course of each treatment fraction. The root mean square error (RMSE) was found for each treatment fraction to compare the 


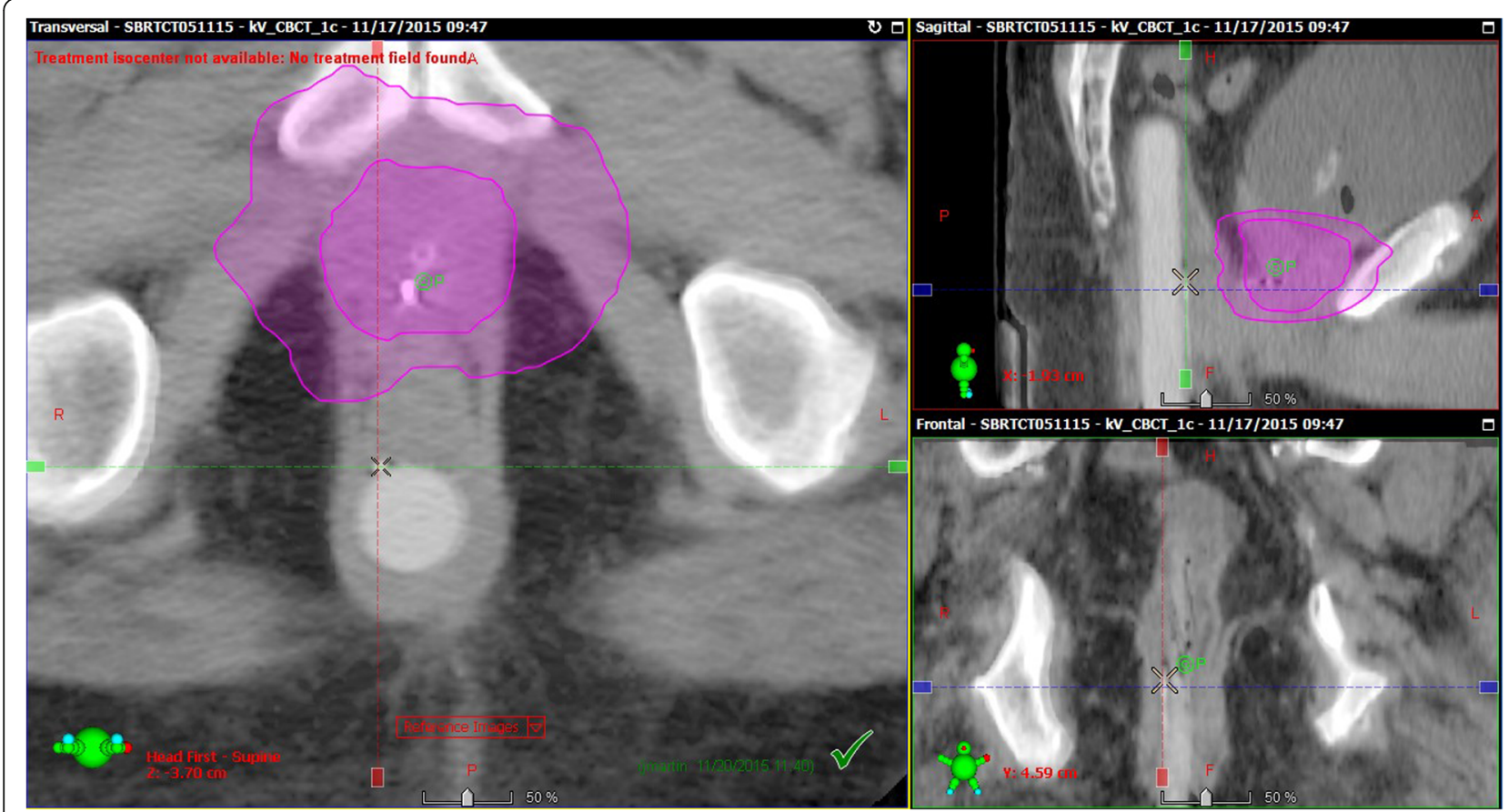

Fig. 4 Marker at MOSkin location on CBCT scan taken following a patient treatment. The CTV and 50\% isodose regions appear in purple

MOSkin measured dose with the planning system dose across the whole treatment time. The RMSE value was normalised to the final planning system dose for each fraction to enable comparison between different patient plans.

\section{Results}

1. Calibration and Testing

The average calibration factor obtained for the dual MOSkins used for phantom and patient treatments was $2.30 \pm 0.07 \mathrm{mV} / \mathrm{cGy}$. The maximum range of
MOSkin responses for dose rates varying from 100 $\mathrm{MU} / \mathrm{min}$ to $600 \mathrm{MU} / \mathrm{min}$ was $\pm 2.6 \%$ for all dose rates measured. When varying the dose delivered, all MOSkin readings were within $\pm 2.0 \%$, indicating a linear response to dose.

The results of the angular dependence testing appear in Fig. 5. The error bars are one standard deviation from the mean of two measurements taken at each angle. The MOSkin readings were normalised to the average MOSkin response for angles from 0 to $330^{\circ}$. All measurements were within $\pm 2.9 \%$ of the average MOSkin reading.

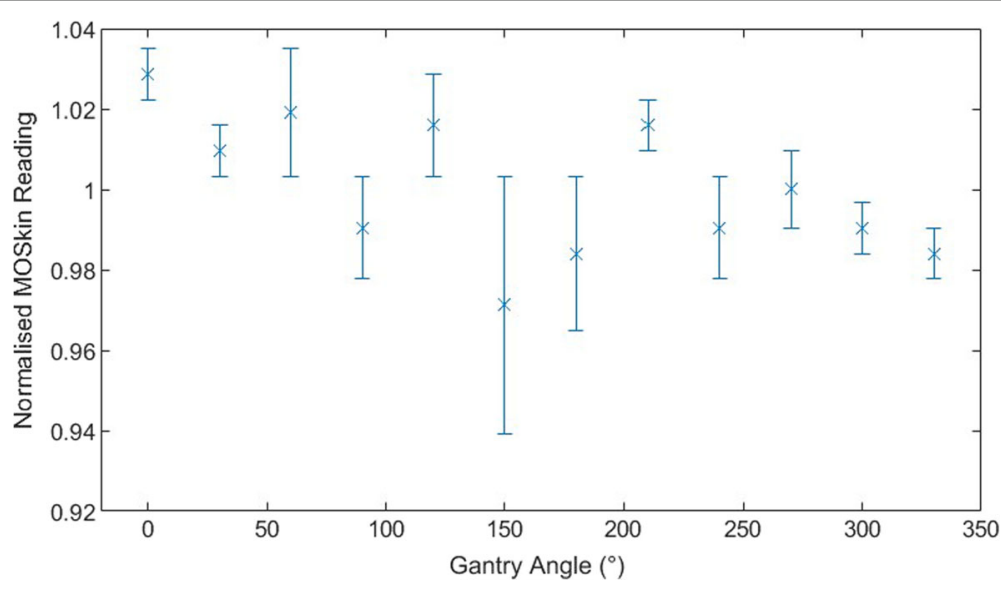

Fig. 5 Angular dependence results for dual MOSkin detector 
2. Phantom Study

The results of the measurements performed in the anthropomorphic phantom appear in Table 1 . The final dose difference was obtained by calculating the cumulative measured dose minus the final planned dose.

Figure 6 presents the dose measured by the dual MOSkin inserted into the anthropomorphic phantom and the planned dose at the MOSkin location.

3. Patient Results

Table 2 presents the normalised RMSE as a percentage of final planned dose for each patient treatment. RMSE comparison was performed so that the practicality of using MOSkin detectors to validate dose delivery in real time could be assessed. RMSE compares the measured and planned doses across the entire treatment delivery. The position of the MOSkin in fraction one was an average of $1.08 \pm 0.66 \mathrm{~cm}$ from the position of the MOSkin during fraction 2.

The average RMSE difference between the measured and planned system doses determined over all fractions measured in this study was $9.7 \%$ with a standard deviation of $3.6 \%$. The cumulative MOSkin reading was lower than the total planned dose for $64 \%$ of the arcs measured. This is illustrated in Fig. 7, which shows the cumulative measured dose minus the total planned dose as a percentage of total planned dose for each patient arc. The average difference between the final measured and final planned doses for all arcs measured was $-3.4 \%$ of the final planned dose, with a standard deviation of $10.3 \%$. The average distance to agreement from the MOSkin location on the plan was $0.14 \pm 0.11 \mathrm{~cm}$ across all patients, with a maximum distance to agreement of $0.47 \mathrm{~cm}$.

Figure 8 shows the comparison of measured and planned dose for a patient treated using a $6 \mathrm{MV}$ beam, so the plan was automatically split into subarcs of $5.2^{\circ}$ in the Eclipse planning system.

\section{Discussion}

MOSkin detectors attached to a Rectafix were used to measure anterior rectal wall dose in real time during prostate SBRT boost treatments. Measured doses were compared with planned doses extracted at the position of the MOSkin post treatment. Small modifications to

Table 1 Comparison of measured and planned doses for dual MOSkin measurements in an anthropomorphic phantom

\begin{tabular}{lccc}
\hline $\begin{array}{l}\text { Treatment } \\
\text { Arc }\end{array}$ & $\begin{array}{l}\text { Normalised RMSE (\% of } \\
\text { final planned dose) }\end{array}$ & $\begin{array}{l}\text { Total dose } \\
\text { difference (cGy) }\end{array}$ & $\begin{array}{l}\text { Final dose } \\
\text { difference (\%) }\end{array}$ \\
\hline Arc 1 & 3.3 & 1.1 & 2.4 \\
Arc 2 & 2.1 & -2.7 & -6.4 \\
\hline
\end{tabular}

the method used in this study could allow the delivered dose to be compared to the planned dose in real time during treatment, allowing for patient repositioning if the measured dose exceeds a preselected dose level during treatment.

The angular dependence measurements for the MOSkin performed in this study found a variation of $\pm 2.9 \%$ in MOSkin dose measurements taken every $30^{\circ}$ of rotation. These results are in reasonable agreement with those measured in a previous study using dual MOSkins by Hardcastle et al., [20] who found a variation of $\pm 2.5 \%$ in readings performed in rotation increments of $30^{\circ}$. The discrepancy in these values may be due to differing geometry in the phantom setup or statistical fluctuations.

Uncertainty in the position of the MOSkin detector and verification point are major sources of discrepancy, as the detector is placed in a high dose gradient region during treatment. Across the rectal wall, the dose can decrease by $4.3 \%$ over a distance of $1 \mathrm{~mm}$, as measured in the treatment planning system, meaning that a small discrepancy in the positioning of the verification point in the plan could mean a large difference in the dose exported from the planning system. While the MOSkin is visible on CBCT scans, precisely placing the verification point on the active area of the MOSkin is difficult, as the detector appears as a long strip with no delineation between the active area and the tail of the MOSkin, and the CBCT resolution makes it difficult to discern the exact location of the end of the detector. Patient shifts occurred between treatment arcs in nearly all cases measured, and patient anatomy in the area of the prostate is mobile, therefore, the verification point selected from the end of treatment CBCT does not indicate the location of the MOSkin detector throughout the entire course of treatment. The average difference between the MOSkin location

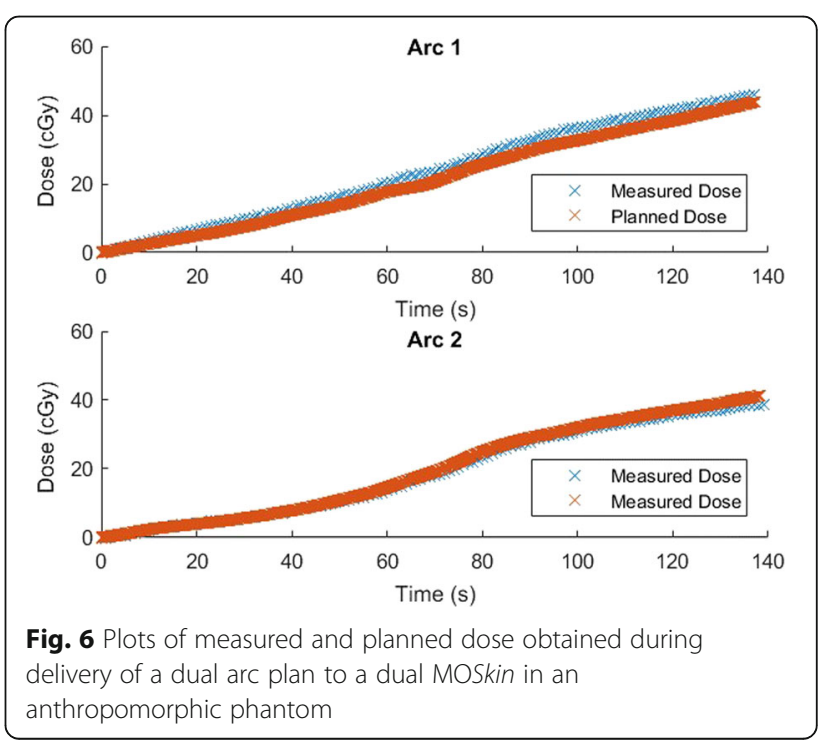


Table 2 Normalised RMSE comparison of measured and planned doses for each treatment fraction

\begin{tabular}{lll}
\hline Patient & $\begin{array}{l}\text { Normalised RMSE Fraction } \\
1 \text { (\% of final planned dose) }\end{array}$ & $\begin{array}{l}\text { Normalised RMSE Fraction } \\
2 \text { (\% of final planned dose) }\end{array}$ \\
\hline 1 & 8.9 & 13.2 \\
2 & 11.8 & 8.4 \\
3 & 11.4 & 13.9 \\
4 & 7.6 & 17.3 \\
5 & 5.3 & 8.5 \\
6 & 9.3 & 15.0 \\
7 & 15.0 & - \\
8 & 4.6 & 6.6 \\
9 & 7.0 & 15.5 \\
10 & 8.4 & 6.5 \\
11 & 6.4 & 6.7 \\
12 & 8.7 & 7.8 \\
Average & 9.7 & \\
Standard & 3.6 & \\
deviation & & \\
\hline
\end{tabular}

on the pre-treatment $\mathrm{CBCT}$ and the post-treatment $\mathrm{CBCT}$ was $0.55 \pm 0.47 \mathrm{~cm}$ which could result in a dose difference in that location of over $20 \%$. The average distance to agreement of $0.14 \pm 0.11 \mathrm{~cm}$ is well within this change in MOSkin location, indicating that the dose discrepancies likely result from the changing position of the MOSkin throughout the treatment due to patient motion and repositioning based on imaging. No fluctuations in detector signal stability were observed due to patient motion.

Previous studies using MOSkins to measure dose in phantoms $[14,16]$ during brachytherapy reported better

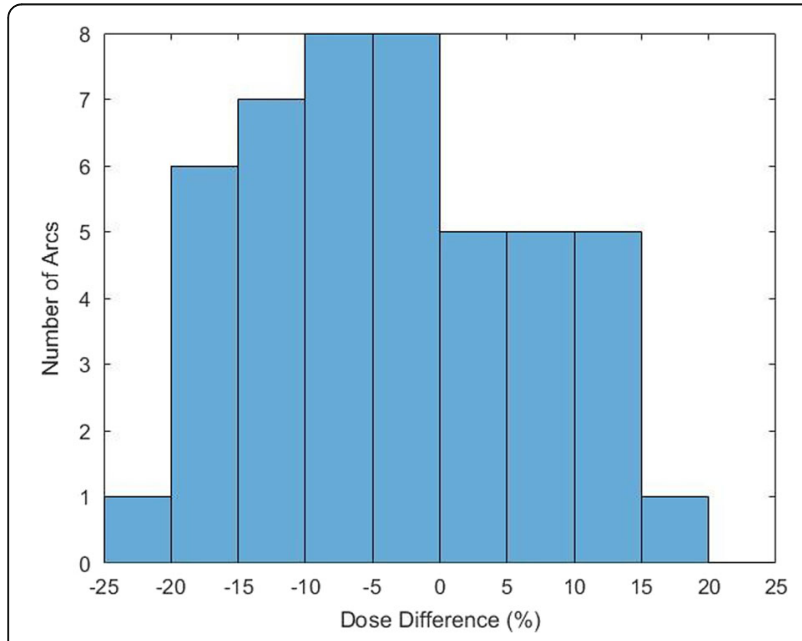

Fig. 7 Comparison of total planned dose and cumulative measured dose per arc. The dose difference was calculated by determining the cumulative measured dose minus the total planned dose for each treatment arc agreement between total measured and planned doses than the patient measurements reported here, as did a phantom study of rectal wall dose measurement during tomotherapy [15]. A phantom study using plastic scintillation detectors attached to endorectal balloons found agreement between measured and planned doses to within $0.5 \%$, [13] and plastic scintillation detectors measured rectal wall dose in patients during EBRT with the mean difference between measured and planned doses for each patient ranging from -3.3 to $3.3 \%$ [17]. Measurements taken using MOSkins in patients during brachytherapy treatments found an average discrepancy between measured and planned doses of $6.3 \%$ with a standard deviation of $4.7 \%$ [18]. The results of the in-patient measurements reported here give a closer average agreement between measured and planned doses than the previous MOSkin study, 3.6\%, with a larger standard deviation of $10.3 \%$.

To adapt the method used here to allow for comparison of measured and planned dose in real time during treatment, Rectafix and MOSkin positioning would need to be consistent at simulation and for each fraction. At present, Rectafix positioning is guided by patient tolerance on the day of treatment. Preparation prior to treatment would require less than half an hour of work per patient to calibrate the MOSkin detectors and export the planned dose at the location of the MOSkin on the simulation CT. The planned dose with time could then be imported into the MOSkin readout software prior to treatment. The delivered dose could then be monitored in real time and adjustments made if the measured dose differed from the planned dose by a predetermined amount. Without these adjustments, the dose tolerance for any interruptions would need to be large, as small differences in the MOSkin position result in large dose differences.

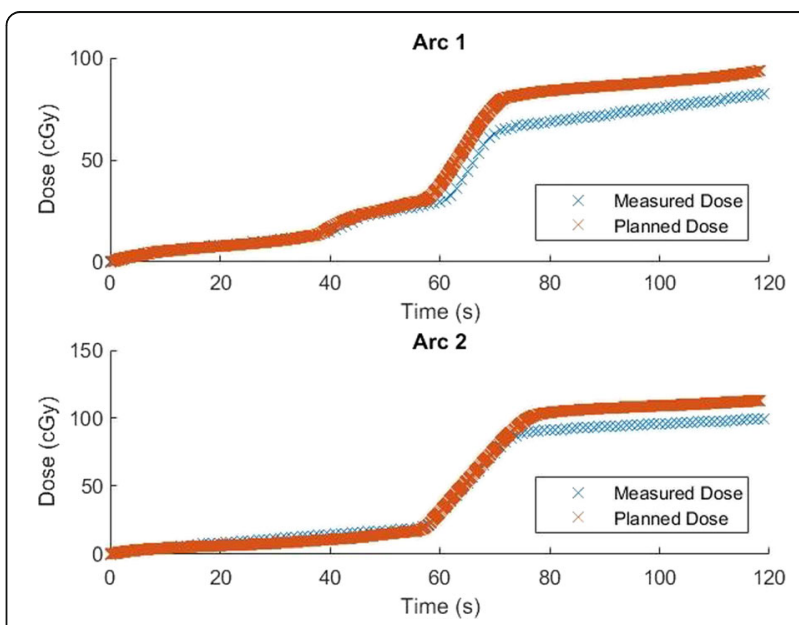

Fig. 8 Comparison of planned and MOSkin measured dose to the anterior rectal wall as a function of time for each arc of a $9.5 \mathrm{~Gy}$ prostate SBRT boost treatment delivered with a $6 \mathrm{X}$ flattened beam. The planned dose has been exported in sub-arcs of $5.2^{\circ}$ 


\section{Conclusions}

MOSkin detectors were used to measure dose to the anterior rectal wall in real time during prostate SBRT boost treatments. The average RMSE dose difference over the entire course of all arcs measured during this study was 9.7\% with a standard deviation of $3.6 \%$. Small modifications to the method presented here would allow MOSkins to provide a dose alarm during SBRT treatments to avoid increased dose to the rectum in the case of delivery errors.

\section{Abbreviations}

$\mathrm{CBCT}$ : Cone beam computed tomography; EBRT: External beam radiation therapy; HDR: High dose rate; RDD: Rectal displacement device; RMSE: Root mean square error; SBRT: Stereotactic body radiation therapy;

VMAT: Volumetric modulated arc therapy

\section{Acknowledgements}

$\mathrm{KL}$ is the recipient of an Australian Postgraduate Award.

\section{Funding}

The authors gratefully acknowledge research funding support from Cancer Council NSW Grant RG11-05.

\section{Availability of data and materials}

The datasets analysed during the current study are available from the corresponding author on reasonable request.

\section{Authors' contributions}

$\mathrm{KL}, \mathrm{PG}, \mathrm{DC}, \mathrm{AW}, \mathrm{JM}$ and DJO made substantial contributions to experimental design and analysis. $\mathrm{KL}, \mathrm{LW}, \mathrm{MR}$ and $\mathrm{PH}$ performed the data acquisition. $\mathrm{KL}$ performed the data analysis. KL drafted the manuscript. DC, DJO, PG and JMM critically revised the manuscript. All authors read and approved the version to be published.

\section{Competing interests}

The authors declare that they have no competing interests.

\section{Consent for publication}

Not applicable.

\section{Ethics approval and consent to participate}

This study was approved by the Hunter New England Human Research Ethics Committee. The reference number is 14/08/20/3.01.

\section{Author details}

${ }^{1}$ University of Newcastle, University Drive, Callaghan 2308, NSW, Australia. ${ }^{2}$ Calvary Mater Newcastle, Cnr Edith and Platt Streets, Waratah 2298, NSW, Australia. ${ }^{3}$ University of Wollongong, Wollongong 2522, NSW, Australia.

Received: 21 July 2016 Accepted: 10 February 2017

Published online: 27 February 2017

\section{References}

1. Vogelius R, Bentzen SM. Meta-analysis of the alpha/beta ratio for prostate cancer in the presence of an overall time factor: bad news, good news, or no news? Int J Radiat Oncol Biol Phys. 2013;85:89-94.

2. Williams G, Taylor JM, Liu N. Use of individual fraction size data from 3756 patients to directly determine the alpha/beta ratio of prostate cancer. Int J Radiat Oncol Biol Phys. 2007;68:24-33.

3. Hoskin PJ, Motohashi K, Bownes P, Bryant L, Ostler P. High dose rate brachytherapy in combination with external beam radiotherapy in the radical treatment of prostate cancer: initial results of a randomised phase three trial. Radiother Oncol. 2007;84:114-20.

4. Khor R, Duchesne G, Tai K-H, Foroudi F, Chander S, Van Dyk S, Garth M, Williams S. Direct 2-arm comparison shows benefit of high-dose-rate brachytherapy boost vs external beam radiation therapy alone for prostate cancer. Int J Radiat Oncol Biol Phys. 2013;85:679-85.
5. Zwahlen DR, Andrianopoulos N, Matheson B, Duchesne GM, Millar JL. Highdose-rate brachytherapy in combination with conformal external beam radiotherapy in the treatment of prostate cancer. Brachytherapy. 2010;9:27-35.

6. Oermann EK, Slack RS, Hanscom HN, Lei S, Suy S, Park HU, Kim JS, Sherer BA, Collins BT, Satinsky AN, Harter KW, Batipps GP, Constantinople NL, Dejter SW, Maxted WC, Regan JB, Pahira JJ, McGeagh KG, Jha RC, Dawson NA, Dritschilo A, Lynch JH, Collins SP. A pilot study of intensity modulated radiation therapy with hypofractionated stereotactic body radiation therapy (SBRT) boost in the treatment of intermediate- to high-risk prostate cancer. Technol Cancer Res Treat. 2010;9:453-62.

7. Jabbari S, Weinberg VK, Kaprealian T, Hsu IC, Ma L, Chuang C, Descovich M, Shiao S, Shinohara K, Roach lii M, Gottschalk AR. Stereotactic body radiotherapy as monotherapy or post-external beam radiotherapy boost for prostate cancer: technique, early toxicity, and PSA response. Int J Radiat Oncol Biol Phys. 2012;82:228-34

8. Katz AJ, Santoro M, Ashley R, Diblasio F, Witten M. Stereotactic body radiotherapy as boost for organ-confined prostate cancer. Technol Cancer Res Treat. 2010;9:575-82

9. Xie Y, Djajaputra D, King CR, Hossain S, Ma L, Xing L. Intrafractional motion of the prostate during hypofractionated radiotherapy. Int J Radiat Oncol Biol Phys. 2008;72:236-46.

10. Hanks GE, Schultheiss TE, Hanlon AL, Hunt M, Lee WR, Epstein BE, Coia LR. Optimization of conformal radiation treatment of prostate cancer: report of a dose escalation study. Int J Radiat Oncol Biol Phys. 1997;37:543-50.

11. Valdagni $R$, Rancati $T$. Reducing rectal injury during external beam radiotherapy for prostate cancer. Nat Rev Urol. 2013;10:345-57.

12. Isacsson U, Nilsson K, Asplund S, Morhed E, Montelius A, Turesson I. A method to separate the rectum from the prostate during proton beam radiotherapy of prostate cancer patients. Acta Oncol. 2010;49:500-5.

13. Archambault L, Briere TM, Pönisch F, Beaulieu L, Kuban DA, Lee A, Beddar S. Toward a Real-Time In Vivo Dosimetry System Using Plastic Scintillation Detectors. Int J Radiat Oncol Biol Phys. 2010;78:280-7.

14. Gambarini G, Carrara M, Tenconi C, Mantaut N, Borroni M, Cutajar D, Petasecca M, Fuduli I, Lerch M, Pignoli E, Rosenfeld A. Online in vivo dosimetry in high dose rate prostate brchytherapy with MOSkin detectors: in phantom feasibility study. Appl Radiat Isot. 2014;83:222-6.

15. Alnaghy SJ, Deshpande S, Cutajar DL, Berk K, Metcalfe P, Rosenfeld AB. In vivo endorectal dosimetry of prostate tomotherapy using dual MOSkin detectors. J Appl Clin Med Phys. 2015;16:5113.

16. Tenconi C, Carrara M, Borroni M, Cerrotta A, Cutajar D, Petasecca M, Lerch M, Bucci J, Gambarini G, Pignoli E, Rosenfeld A. TRUS-probe integrated MOSkin detectors for rectal wall in vivo dosimetry in HDR brachytherapy: in phantom feasibility study. Radiat Meas. 2014;71:379-83.

17. Wootton $L$, Kudchadker $R$, Lee $A$, Beddar S. Real-time in vivo rectal wall dosimetry using plastic scintillation detectors for patients with prostate cancer. Phys Med Biol. 2014;59:647-60.

18. Carrara M, Tenconi C, Rossi G, Borroni M, Cerrotta A, Grisotto S, Cusumano D, Pappalardi B, Cutajar D, Petasecca M, Lerch M, Gambarini G, Fallai C, Rosenfeld A, Pignoli E. In vivo rectal wall measurements during HDR prostate brachytherapy with MOSkin dosimeters integrated on a trans-rectal US probe: comparison with planned and reconstructed doses. Radiother Oncol. 2016;118:148-53.

19. Kwan IS, Rosenfeld AB, Qi ZY, Wilkinson D, Lerch MLF, Cutajar DL, SafaviNaeni M, Butson M, Bucci JA, Chin Y, Perevertaylo VL. Skin dosimetry with new MOSFET detectors. Radiat Meas. 2008;43:929-32.

20. Hardcastle N, Cutajar D, Metcalfe P, Lerch MLF, Perevertaylo VL, Tome WA, Rosenfeld A. In vivo real-time rectal wall dosimetry for prostate radiotherapy. Phys Med Biol. 2010;55:3859-71.

21. Cheung T, Martin JB, Peter KNY. Effects of temperature variation on MOSFET dosimetry. Phys Med Biol. 2004;49:N191.

22. Rosenfeld AB, Siegbahn EA, Brauer-Krish E, Holmes-Siedle A, Lerch MLF, Bravin A, Cornelius IM, Takacs GJ, Painuly N, Nettelback H, Kron T. Edge-on face-to-face MOSFET for synchrotron microbeam dosimetry: MC modeling. IEEE Trans Nucl Sci. 2005:52:2562-9. 